\title{
Preparation and characterization of nanosilica from rice husk ash by chemical treatment combined with calcination
}

\author{
Le Nghiem Anh Tuan ${ }^{1,2}$, Lai Thi Kim Dung ${ }^{1}$, Le Doan Thanh $\mathrm{Ha}^{1}$, \\ Nguyen Quoc Hien', Dang Van Phu ${ }^{2,3}$, Bui Duy Du, ${ }^{1,2 *}$ \\ ${ }^{1}$ Institute of Applied Materials Science, Vietnam Academy of Science and Technology \\ ${ }^{2}$ Graduate University of Science and Technology, Vietnam Academy of Science and Technology \\ ${ }^{3}$ Research and Development Center for Radiation Technology, Vietnam Atomic Energy Institute
}

Received 9 December 2016; Accepted for publication 28 August 2017

\begin{abstract}
This work presented the results of the study on synthesis and characterization of nanosilica from rice husk ash (RHA). Nanosilica was obtained by calcination of $\mathrm{HCl}$ treated RHA at $700{ }^{\circ} \mathrm{C}$ for $2 \mathrm{~h}$. X-ray diffraction (XRD) pattern showed only one peak at $2 \theta \sim 22^{\circ}$ confirming the amorphous structure of nanosilica. The chemical composition assessed by energy dispersive X-ray spectroscopy (EDX) showed that the obtained nanosilica was of high purity. TEM image measured by transmission electron microscopy (TEM) revealed that the obtained nanosilica was in spherical morphology with the average diameter of about $45.5 \pm 7.2 \mathrm{~nm}$. The particle size distribution of nanosilica determined by dynamic laser scattering (DLS) was of Gaussian mode. The FTIR spectrum indicated the presence of silanol and siloxane groups in nanosilica. Thus, the synthesized nanosilica can be used for application in different fields.
\end{abstract}

Keywords. Rice husk ash, nanosilica, amorphous.

\section{INTRODUCTION}

Nanotechnology has attracted considerable scientific interest due to the potential uses of the nanomaterials and nanocomposite. There are several reports on synthesized nanosilica used in vegetable oil refining, as medicines, detergents, adhesives, ceramics, and pesticide [1, 2]. Tetraethoxysilane (TEOS) and tetramethoxysilane (TMOS) have been usually used as the silica source to produce nanosilica [1]. However, these chemical sources are rather expensive and toxic. Rice husk ash (RHA), rich in silica of about 83-90\% is one of the waste products [1, 3]. Typically, the major remaining inorganic component of Vietnamese RHA is $\mathrm{SiO}_{2}$ (96.15\%), along with some minor inorganic constituents including aluminium oxide $(0.48 \%)$, iron oxide $(0.15 \%)$, calcium oxide $(0.73 \%)$, magnesium oxide $(0.55 \%)$, sodium oxide $(0.12 \%)$, potassium oxide $(0.39 \%)$, and a loss of ignition $(1.43 \%)$ [4]. Various methods for preparing nanosilica from rice husk $(\mathrm{RH})$ have been reported in the literature, such as sol-gel $[4,5]$, precipitation [6-8], and pyrolysis method [3, 9-14]. The world's most researching synthesis of nanosilica from $\mathrm{RH}$ is that metal oxides were removed from $\mathrm{RH}$ by treatment with acid, then silica was extracted by alkaline $(\mathrm{NaOH})$, precipitated with acid and finally calcined at high temperature to obtain nanosilica $[3,5,11]$. The reaction processes are described as follows:

$$
\begin{aligned}
& \mathrm{SiO}_{2} \text { (ash) }+2 \mathrm{NaOH} \rightarrow \mathrm{Na}_{2} \mathrm{SiO}_{3}+\mathrm{H}_{2} \mathrm{O} \\
& \mathrm{Na}_{2} \mathrm{SiO}_{3}+\mathrm{H}_{2} \mathrm{SO}_{4} \rightarrow \mathrm{SiO}_{2} \downarrow+\mathrm{Na}_{2} \mathrm{SO}_{4}+\mathrm{H}_{2} \mathrm{O}
\end{aligned}
$$

Besides, many authors also studied a simple synthesis process that metal oxides were removed from $\mathrm{RH}$ by treatment with acid $\left(\mathrm{HCl}, \mathrm{H}_{2} \mathrm{SO}_{4}\right.$, organic acids) and then nanosilica was obtained by calcination of acid treated $\mathrm{RH}$ at temperature from 500 to $800{ }^{\circ} \mathrm{C}[9,12,13,15]$. There is also a recyclable method to obtain silica from RHA by using $\mathrm{NH}_{4} \mathrm{~F}$ that dissolved $\mathrm{SiO}_{2}$ and precipitated $\mathrm{SiO}_{2}$ by $\mathrm{NH}_{3}$ [16]. The silica powder was obtained by the following reactions:

$$
\begin{aligned}
& 6 \mathrm{NH}_{4} \mathrm{~F}+\mathrm{SiO}_{2}(\mathrm{RHA}) \rightarrow \\
& \quad\left(\mathrm{NH}_{4}\right)_{2} \mathrm{SiF}_{6}+4 \mathrm{NH}_{3}+2 \mathrm{H}_{2} \mathrm{O} \\
& \left(\mathrm{NH}_{4}\right)_{2} \mathrm{SiF}_{6}+4 \mathrm{NH}_{3}+(\mathrm{n}+2) \mathrm{H}_{2} \mathrm{O} \rightarrow \\
& \quad 6 \mathrm{NH}_{4} \mathrm{~F}+\mathrm{SiO}_{2} \downarrow+\mathrm{nH}_{2} \mathrm{O}
\end{aligned}
$$

In Vietnam, Le et al. studied on synthesis of nanosilica by the sol-gel method [4]. Silica from RHA was extracted by sodium hydroxide to produce 
sodium silicate solution and then silica was precipitated from sodium silicate solution by adding $\mathrm{H}_{2} \mathrm{SO}_{4}$ in the mixture of water/butanol and finally the precipitate was calcinated at $550{ }^{\circ} \mathrm{C}$ for $4 \mathrm{~h}$ in atmospheric condition to obtain nanosilica. Nguyen et al. also prepared nanosilica from RHA by precipitation method [8]. Silica was extracted by sodium hydroxide solution and after that silica was precipitated with $\mathrm{HCl}$ solution.

In this paper, we present a process for synthesizing nanosilica from RHA for us as an agent for growth stimulation and disease resistance for plants. Nanosilica was synthesized from RHA by two steps. In the first step, metal oxide impurities were removed from RHA by treatment with acid. In the second step, the acid treated RHA was calcined at $700{ }^{\circ} \mathrm{C}$.

\section{EXPERIMENTAL}

\subsection{Materials}

Rice husk from Dong Nai province, Vietnam was burned in open environment to collect RHA. $\mathrm{HCl}$ acid was purchased from Merck, Germany. Distilled water was used in all experiments.

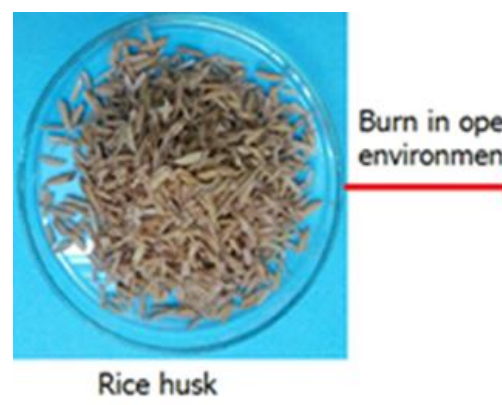

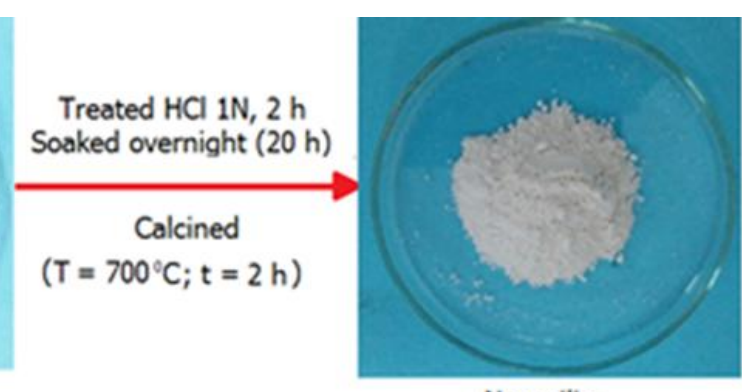

Nanosilica

Figure 1: Schematic illustration of the synthesis mechanism for nanosilica powder from rice husk ash

\subsection{Preparation of nanosilica}

$5.0 \mathrm{~g}$ of RHA was stirred with $30 \mathrm{~mL}$ of $1 \mathrm{~N} \mathrm{HCl}$ for $2 \mathrm{~h}$ at $80{ }^{\circ} \mathrm{C}$ and let standing overnight to remove metal ions in RHA [3, 12]. Then, acid treated RHA was filtered, washed with distilled water and dried at $110^{\circ} \mathrm{C}$ in an electric oven. The acid treated RHA was then calcinated in a programmable furnace (Nabertherm $\mathrm{GmbH}$, Germany) at $700{ }^{\circ} \mathrm{C}$ for $2 \mathrm{~h}$ to obtain nanosilica $[3,9,14]$. The schematic illustration of the synthesis process of nanosilica was described in Fig. 1.

\subsection{Characterization of nanosilica}

The functional groups of the $\mathrm{SiO}_{2}$ nanoparticles were analyzed by FT-IR technique. Spectral-grade $\mathrm{KBr}$ powder was mixed with nanosilica at a weight ratio of $2 \mathrm{mg} \mathrm{SiO}_{2}: 200 \mathrm{mg} \mathrm{KBr}$ in an agate mortar. The mixture powder was pressed into pellets with a diameter of $13 \mathrm{~mm}$ and thickness of $0.5 \mathrm{~mm}$. The infrared (IR) spectrum of nanosilica was measured by using FTIR spectroscopy (FT-IR 8400S, Shimadzu) over the wavenumber range from 4000 to $400 \mathrm{~cm}^{-1}$.

X-ray diffractometer (D8 Avance Bruker, Germany) was used to determine the amorphous phase of nanosilica. The XRD pattern was obtained by using $\mathrm{CuK} \alpha$ as a radiation source $(\lambda=1.5405 \AA)$ operating under a constant current of $30 \mathrm{~mA}$ at 40 $\mathrm{kV}$ with a diffraction angle $(2 \theta)$ scan range from 5 to $80^{\circ}$.

The morphology and particle size of the nanosilica were measured using a transmission electron microscope (TEM) (JEM1010, JEOL, Japan). Silica particle size was statistically calculated from TEM image by Photoshop CS6 and Microsoft EXCEL 2010 softwares.

Dynamic light scattering (DLS) analysis of nanosilica was also performed using a Nano-Particle Size Analyzer (HORIBA, LB550, Japan).

The chemical composition of nanosilica was assessed by energy dispersive $\mathrm{X}$-ray spectroscopy (EDX) on a JEOL 6610 LA.

\section{RESULTS AND DISCUSSION}

The inorganic metallic ions impurities in RHA were separated into the liquid by $\mathrm{HCl}$ leaching RHA suspension. After acid treatment, the organic constituents and silica remained as solid ingredients. The chemical reaction along with the hightemperature calcination for the preparation of nanosilica powder can be described as follows: 


$$
\mathrm{RHA}+\mathrm{HCl} \stackrel{80^{\circ} \mathrm{C}(2 \mathrm{~h}) \text {, soaked overnight }}{\longrightarrow} \mathrm{SiO}_{2}(\mathrm{Ash})+\mathrm{MCl}+\mathrm{H}_{2} \mathrm{O}
$$

Where M: Na; K; Ca; Fe; Al, Mg,...

$$
\mathrm{SiO}_{2} \text { (Ash) } \stackrel{700{ }^{\circ} \mathrm{C}(2 \mathrm{~h})}{\longrightarrow} \mathrm{SiO}_{2} \text { (nanosilica powder) }
$$

During the high temperature calcinating process, all of the organic constituents were burned out. Finally, white powder nanosilica was obtained (Fig. $1)$.

The chemical composition of silica analyzed by EDX was presented in Fig. 2. As shown in Fig. 2, nanosilica contains only silicon and oxygen with weight ratio of about 1:1.5. This result confirmed that the obtained nanosilica is in good stoichiometric ratio and high purity. The elements such as $\mathrm{Ca}, \mathrm{K}$, $\mathrm{Na}, \mathrm{Mg}, \mathrm{Fe}, \mathrm{Al}$, and $\mathrm{Mg}$.that were reported in composition of RHA were not detected in nanosilica obtained by calcination of acid treatment of RHA [3, $5,8,12,14]$.

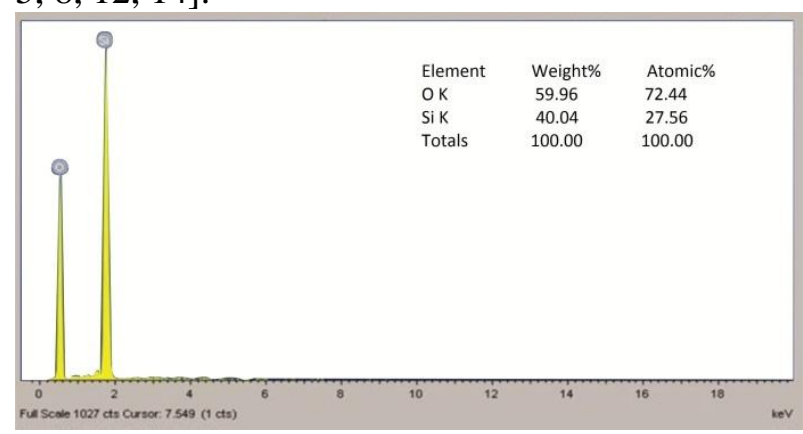

Figure 2: EDX spectrometric data of nanosilica produced from RHA

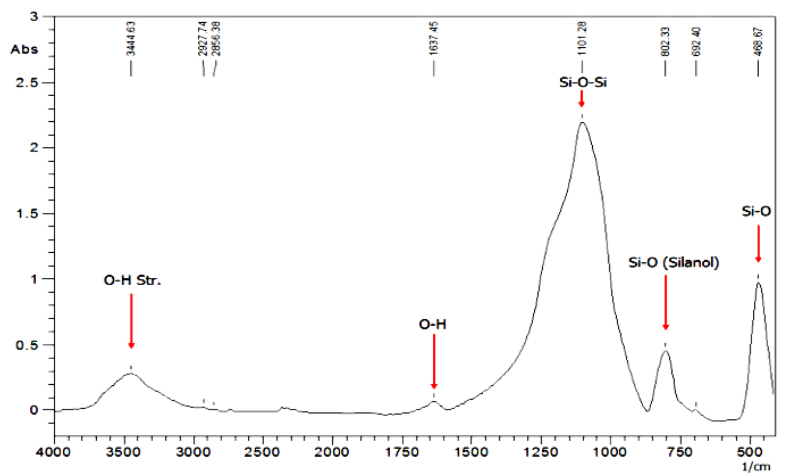

Figure 3: FT-IR spectrum of nanosilica from RHA

The FT-IR spectrum and FT-IR spectral data of nanosilica were shown in Fig. 3 and Table 1, respectively. The peaks at 468 and $802 \mathrm{~cm}^{-1}$ assigned to the rocking bond and symmetric bond vibrations of the $\mathrm{Si}-\mathrm{O}$ (silanol), respectively. The peak at $1101 \mathrm{~cm}^{-1}$ is related to the vibrational stretching of asymmetric $\mathrm{Si}-\mathrm{O}-\mathrm{Si}$ in $\mathrm{S} 1 \mathrm{O} 4$ tetrahedron [3], showing a stoichiometric silicon dioxide $\left(\mathrm{SiO}_{2}\right)$ structure. The $\mathrm{O}-\mathrm{H}$ bending and stretching vibration modes also appeared in the absorption band region at 3444 and at $1637 \mathrm{~cm}^{-1}$, respectively.

Table 1: FT-IR spectral data of nanosilica from RHA

\begin{tabular}{|c|c|c|c|}
\hline Frequency $\left(\mathrm{cm}^{-1}\right)$ & Position assignment & Literature value & References \\
\hline 468 & Si-O bond rocking & $438-475$ & {$[3,4,6,8,10,11,14,17]$} \\
\hline 802 & Symmetric Si-O bending (silanol) & $796-805$ & {$[3,4,6,10,11,14,17]$} \\
\hline 1101 & $\begin{array}{c}\text { Asymmetric Si-O-Si stretching in } \\
\text { S1O4 tetrahedron }\end{array}$ & $1050-1150$ & {$[3,4,6,10,11,14,17]$} \\
\hline 1637 & O-H bending & $1633-1643$ & {$[3,4,8,10]$} \\
\hline 3444 & O-H stretching and adsorbed water & $3437-3456$ & {$[3,4,8,10,11,17]$} \\
\hline
\end{tabular}

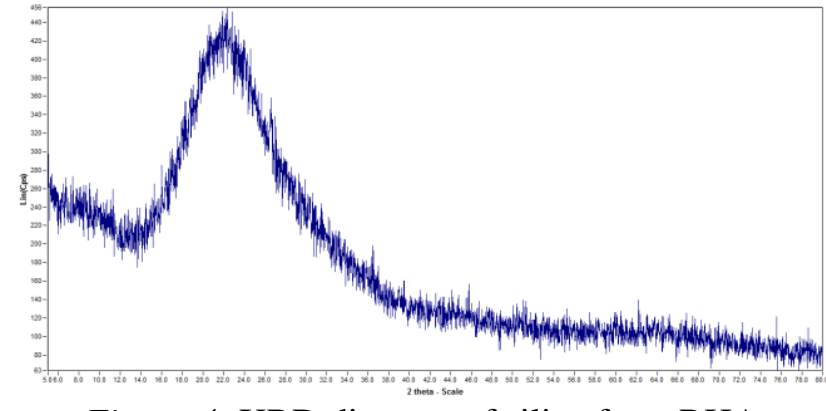

Figure 4: XRD diagram of silica from RHA
The XRD pattern of the obtained nanosilica in Fig. 4 showed only a single peak at $2 \theta \sim 22^{\circ}$ which confirmed the amorphous structure of nanosilica [3, $4,6,11,14-18]$.

The TEM image and particle size distribution of nanosilica were shown in Fig. 5. It can be observed from Fig. 5 that the obtained nanosilica was almost in spherical morphology with the average diameter of about $45.5 \pm 7.2 \mathrm{~nm}$ and the particle size distribution was in a fairly narrow range of 30-60 
$\mathrm{nm}$. The size of nanosilica obtained in this work was almost the same that of nanosilica synthesized from RH by other authors [3, 6, 8-11].

The size and particle size distribution of prepared nanosilica were also determined by DLS
(Fig. 6). The results showed that particle size of nanosilica was in the range of 200-400 $\mathrm{nm}$ and the distribution was of Gaussian mode. Thus, the size of nanosilica from TEM image seems to be usually smaller than that from DLS [12]
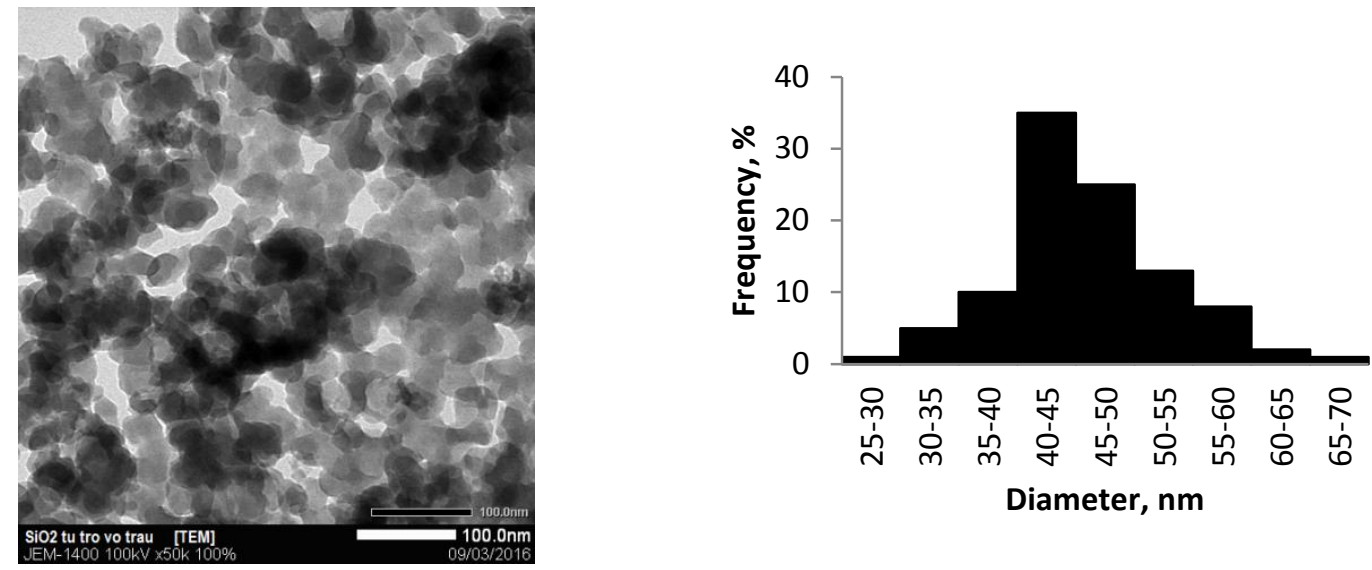

Figure 5: TEM images and distribution of particle size of nanosilica

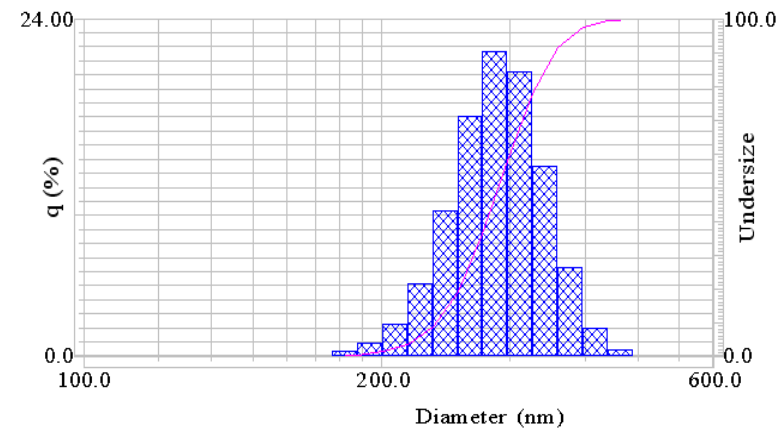

Figure 6: Distribution of particle size by DLS

Based on the results obtained, the method of synthesis of nanosilica from RHA is considered as an economical and suitable method for production of nanosilica on large scale for different applications.

\section{CONCLUSION}

In this study, a source attempt has been made to use cheap RHA to synthesize nanosilica with diameter of about $45.5 \mathrm{~nm}$ and narrow particle size distribution by acid treatment combined with calcination. This method is considered as a suitable and economical method to produce nanosilica on large scale. The obtained nanosilica can be applied in various fields and helps to minimize the concerns of RHA disposal.

Acknowledgments. This research is funded by Vietnam National Foundation for Science and Technology Development (NAFOSTED) under grant number 106-NN.03-2015.84.

\section{REFERENCES}

1. Y. Shen, P. Zhao, Q. Shao. Porous silica and carbon derived materials from rice husk pyrolysis charreview, Microporous and Mesoporous Materials, 188, 46-76 (2014).

2. R. Pode. Potential applications of rice husk ash waste from rice husk biomass power plant, Renewable and Sustainable Energy Reviews, 53, 1468-1485 (2016).

3. S. Sankar, S. K. Sharma, N. Kaur, B. Lee, D. Y. Kim, S. Lee, H. Jung. Biogenerated silica nanoparticles synthesized from sticky, red, and brown rice husk ashes by chemical method, Ceramics International, 42, 4875-4885 (2016).

4. V. H. Le, C. N. H. Thuc, H. H. Thuc. Synthesis of silica nanoparti-cles from Vietnamese rice husk by sol-gel method, Nanoscale Research Letters, 8(1), 5867 (2013).

5. P. Lu and Y. L. Hsieh. Highly pure amorphous silica nano-disks from rice straw, Powder Technology, 225, 149-155 (2012).

6. T. H. Liou and C. C. Yang. Synthesis and surface characteristics of nanosilica produced from alkaliextracted rice husk ash, Materials Science and Engineering B, 176, 521-529 (2011).

7. A. Tadjarodi, M. Haghverdi, V. Mohammadi. Preparation and characterization of nano-porous silica aerogel from rice husk ash by drying at atmospheric pressure, Materials Research Bulletin, 47, 584-2589 (2012).

8. T. T. Nguyen, H. M. P. Nguyen, N. T. T. Ho, T. B. T. Pham, T. K. C. Nguyen, V. N. Le, T. T. Nguyen, X. A. Trinh. Synthesis of $\mathrm{SiO}_{2}$ nanoparticles from rice husk ash by precipitation method, Journal of Science 
VJC, 55(4), 2017

Cantho university-Vietnam, 32, 120-124 (2014).

9. W. Wang, J. C. Martin, X. Fan, A. Han, Z. Luo, L. Sun. Silica nanoparticles and frameworks from rice husk biomass, ACS Applied Materials and Interfaces, 4, 977-981 (2012).

10.E. Rafiee, S. Shahebrahimi, M. Feyzi, M. Shaterzadeh. Optimization of synthesis and characterization of nanosilica produced from rice husk (a common waste material), International Nano Letters, 2, 29 (2012).

11.S. Gu, J. Zhou, Z. Luo, Q. Wang, M. Ni. A detailed study of the effects of pyrolysis temperature and feedstock particle size on the preparation of nanosilica from rice husk, Industrial Crops and Products, 50, 540-549 (2013).

12.A. A. Alshatwi, J. Athinarayanan, V. S. Periasamy. Biocompatibility assessment of rice husk-derived biogenic silica nanoparticles for biomedical applications, Materials Science and Engineering C, 47, 8-16 (2015).

13.G. M. F. Gomes, C. Philipssen, E. K. Bard, L. D. Zen, G. de Souza. Rice husk bubbling fluidized bed combustion for amorphous silica synthesis, Journal of Environmental Chemical Engineering, 4(2), 2278-
Preparation and characterization of nanosilica...

2290 (2016).

14.R. A. Bakar, R. Yahya, S. N. Gan. Production of high purity amorphous silica from rice husk, Procedia Chemistry, 19, 189-195 (2016).

15.S. Gu, J. Zhou, C. Yu, Z. Luo, Q. Wang, Z. Shi. A novel two-staged thermal synthesis method of generating nanosilica from rice husk via pre-pyrolysis combined with calcination, Industrial Crops and Products, 65, 1-6 (2015).

16.X. Ma, B. Zhou, W. Gao, Y. Qu, L. Wang, Z. Wang, Y. Zhu. A recyclable method for production of pure silica from rice hull ash, Powder Technology, 217, 497-501 (2012).

17.H. Sixiao and Y. L. Hsieh. Preparation of activated carbon and silica particles from rice straw, ACS Sustainable Chemistry \& Engineering, 2, 726-734 (2014).

18.P. D. Dung, L. S. Ngoc, N. N. Duy, N. N. Thuy, L. T. M. Truc, B. V. Le, D. V. Phu, N. Q. Hien. Effect of nanosilica from rice husk on the growth enhancement of chili plant (Capsicum frutescens L.), J. Sci. Technol., 54(5), 607-613 (2016).

\section{Corresponding author: Bui Duy Du}

Institute of Applied Materials Science

Vietnam Academy of Science and Technology

No. 1, Mac Dinh Chi Str., District 1, Ho Chi Minh City

E-mail:vina9802@gmail.com; Telephone: 0931797968. 